\title{
PHENOLIC CONTENT, ANTIOXIDANT AND ANTIMICROBIAL POTENTIAL OF MELANOLEUCA MELALEUCA EDIBLE MUSHROOM
}

\author{
M. Sevindik \\ Department of Food Processing, Bahçe Vocational School, Osmaniye Korkut Ata University, Osmaniye, Turkey \\ ORCID: 0000-0001-7223-2220 \\ Corresponding Author's E mail: sevindik27@gmail.com
}

\begin{abstract}
Wild edible mushrooms have been gathered and consumed by humans since ancient times. In addition to their nutritional properties, many mushrooms are known to possess medicinal properties. The present study aimed to determine antioxidant and antimicrobial activities and phenolic content of the wild edible mushroom Melanoleuca melaleuca (Pers.) Murrill. All experiments were performed 5 times, and results expressed as mean \pm standard deviation unless otherwise stated $(\mathrm{p} \leq 0: 05)$. The present study findings demonstrated that the antioxidant potential of the mushroom was high. It was determined that the TAS value of ethanol extract of the M. melaleuca mushroom was $3.393 \pm 0.098 \mathrm{mmol} / \mathrm{L}$, TOS value was $6.460 \pm 0.121 \mu \mathrm{mol} / \mathrm{L}$ and OSI value was $0.190 \pm 0.002$. Furthermore, it was determined that the mushroom extracts were effective against test microorganisms at $25-400 \mu \mathrm{g} / \mathrm{mL}$ concentrations. HPLC scans revealed that the mushroom contained gallic, catechin, p-coumaric, syringic and protocatechuic acids. Thus, it was determined that the mushroom had antioxidant and antimicrobial potential. It was also determined that the mushroom could serve as a natural source for phenolic content.
\end{abstract}

Keywords: Antimicrobial, antioxidant, phenolic content, Melanoleuca melaleuca, wild edible mushroom.

\section{INTRODUCTION}

Reactive oxygen species (ROS) produced by living organisms could be beneficial at low levels. For example, ROS play physiological roles in cellular response to noxia such as defense against infectious agents, and in the functioning of certain cellular signaling systems (Rahman, 2007). However, due to increased ROS levels, damages could occur in cellular structures such as lipids, membranes, proteins and nucleic acids (Rahman, 2007; Pham-Huy et al., 2008). These harmful effects of ROS could be inhibited or reduced by endogenous antioxidants. However, oxidative stress could be observed when endogenous antioxidants are insufficient. Oxidative stress could lead to several chronic and degenerative diseases such as cancer, arthritis, aging, autoimmune, cardiovascular and neurodegenerative diseases. In cases where endogenous antioxidants are insufficient to counteract the effects of ROS, supplementary antioxidants could be beneficial in the prevention or reduction of oxidative stress. Exogenous antioxidants function as free radical scavengers by preventing and repairing the damages caused by the ROS. Thus, they could improve the immune defense and reduce the cancer and degenerative disease risks (Rahman, 2007; Pham-Huy et al., 2008; Nimse and Pal, 2015; Suleman et al., 2019). Natural products with antioxidant activity such as the mushrooms are used as supplementary antioxidants. In recent times, there is an increasing interest in mushrooms used to assist endogenous antioxidants.

Microorganisms are responsible for several diseases globally. While several of these diseases could be easily treated, today, these diseases are becoming difficult to treat due to resistant microorganisms. Therefore, it became necessary to identify and investigate natural products that are effective against resistant microorganisms (Nowacka et al., 2015; Sevindik, 2018). Natural mushrooms are living organisms that have not been investigated adequately. Thus, it is very important to investigate the potential of mushrooms as an antimicrobial agent.

Mushrooms have been used for many purposes in several parts of the world for a long time. They are mainly used as nutrients and for their medicinal properties. They have high protein content, are rich in vitamin B, various minerals and contain almost all essential amino acids. In addition to their nutritional properties, mushrooms are good therapeutic agents. Mushrooms could also provide health benefits as well as their nutritional values. In previous studies, it was reported that mushrooms had significant antioxidant, antibacterial, anticancer, antigenotoxic, antiinflammatory, antifungal and DNA protective effects (Arora et al., 2013; Bal et al., 2019; Meng et al., 2016; Yilmaz et al., 2016; Muszyńska et al., 2018).

Melanoleuca melaleuca (Pers.) Murrill is an edible mushroom. It is prevalent in mixed forests. The 
present study aimed to determine the antioxidant and antimicrobial activities and phenolic content of the wild and edible $M$. melaleuca mushroom.

\section{MATERIALS AND METHODS}

Laboratory Studies: $M$. melaleuca samples investigated in the present study were collected in Oguzeli/Turkey. Mushroom samples were dried at $+40^{\circ} \mathrm{C}$ (Profilo, PFD1350W, Turkey). Ten grams of the pulverized mushroom samples were macerated $(24 \mathrm{~h})$ with $200 \mathrm{~mL}$ ethanol $(\mathrm{EtOH})$ using a magnetic stirrer. They were then concentrated via filtration at $40^{\circ} \mathrm{C}$ using a rotary evaporator.

Phenolic Content Analyses: The phenolic contents of the mushroom were determined with a RPHPLC-DAD system (Shimadzu). The presence of gallic acid, catechin, chlorogenic acid, caffeic acid, epicatechin, syringic acid, coumaric acid, benzoic acid, hydroxybenzoic acid, hesperidin, sinapic acid, protocatechuic acid and rosmarinic acid was investigated. To perform the analysis, the standards of the compounds were injected. Then, standards were scanned in the mushroom sample. Then, the peak resolution and run time was defined for each compound. A DAD detector was utilized in the process. Chromatographic processes were conducted with an Agilent Eclipse XDB-C18 column (250x4.6 mm; id 5 $\mu \mathrm{m})$ at $30^{\circ} \mathrm{C}$ (Caponio et al., 1999).

Antioxidant and Oxidant Analyses: TAS, TOS and OSI values of ethanol extract of $M$. melaleuca mushroom were determined with commercial Rel assay kits TAS and TOS (Rel Assay Kit Diagnostics, Turkey). Analysis was conducted on 5 replicates. Hydrogen peroxide (TOS) and Trolox (TAS) were used as the calibrator. Results are expressed as mmol Trolox equiv./L for TAS and $\mu \mathrm{mol}$ $\mathrm{H}_{2} \mathrm{O}_{2}$ equiv./L for TOS (Erel, 2004, 2005). The following formula was used to calculate the OSI (arbitrary unit: AU) (Erel, 2005).

$$
O \quad \text { (A ) }: \frac{\mathrm{T} \quad\left(\mu \mathrm{mol} \mathrm{H}_{2} \mathrm{O}_{2}\right. \text { equiv./L) }}{T \quad \text { (mmol Trolox equiv./L }) \times 10}
$$

Antimicrobial Analyses: Antimicrobial activity analyses were conducted using the agar dilution method on mushroom extracts. Bacterial strains: Staphylococcus aureus ATCC 29213, S. aureus MRSA ATCC 43300, Enterococcus faecalis ATCC 29212, Escherichia coli ATCC 25922, Pseudomonas aeruginosa ATCC 27853 and Acinetobacter baumannii ATCC 19606. Fungal strains: Candida albicans ATCC 10231, C. krusei ATCC 34135 ATCC 13803 and C. glabrata ATCC 90030. Precultured processes were performed in Muller Hinton Broth for bacteria and RPMI 1640 Broth for fungi. Fungi reference drugs: Fluconazole and Amphotericin B. Bacteria reference drugs: Amikacin, Ampicillin and Ciprofloxacin. Mushroom extracts were tested at 800,
400, 200, 100, 50, 25 and $12.5 \mu \mathrm{g} / \mathrm{mL}$ concentrations. The concentrations were adjusted using distilled water. The lowest concentration that inhibited the bacteria and fungi growth was determined as the minimum inhibitor concentration (MIC) (Bauer et al., 1966; Hindler et al., 1992; CLSI, 2012; EUCAST, 2014; Matuschek et al., 2014; EUCAST, 2015).

Statistical Analysis: All experiments were performed 5 times, and results expressed as mean \pm standard deviation unless otherwise stated. Statistical analysis was performed using Microsoft Excel. Results were considered significant $(\mathrm{P} \leq 0.05)$.

\section{RESULTS AND DISCUSSION}

Phenolic Content: The mushrooms, which have rich organic and inorganic content, synthesize secondary metabolites as a result of metabolic and physiological activities. These secondary metabolites exhibit various biological activities (Zhong and Xiao 2009; Chen and Liu 2017). Thus, it has been seemed worth to study the biological action mechanisms and activities of mushrooms. Also, analysis of the phenolic compounds that are primarily responsible in mushroom research for biological activities is very important. In the present study, phenolic acid content in $M$. melaleuca mushroom was determined. In HPLC analysis, 13 phenolic acids were screened in the mushroom. Analysis findings demonstrated that 5 compounds (Gallic acid, catechin, pcoumaric acid, syringic acid and protocatechuic acid) were present. The analysis findings are presented in Table 1.

Table 1. Phenolic Contents of $M$. melaleuca.

\begin{tabular}{lc}
\hline Phenolic Compound (ppm) & M. melaleuca \\
\hline Gallic Acid & 14.062 \\
Catechin & 5.328 \\
Chlorogenic Acid & none \\
Caffeic Acid & none \\
Epicatechin & none \\
Syringic Acid & 1.251 \\
P-Coumaric Acid & 26.231 \\
Benzoic Acid & none \\
Hydroxybenzoic Acid & none \\
Hesperidin & none \\
Sinapic Acid & none \\
Rosmarinic Acid & none \\
Protocatechuic Acid & 41.820 \\
\hline
\end{tabular}

Previous studies reported that gallic acid is a common and important antioxidant compound found in several natural products (Zanwar et al., 2014). It was also reported that gallic acid exhibits various biological properties such as anticancer, antimicrobial, anti- 
inflammatory, antitumor, anti-neoplastic and anti-diabetic effects (Kroes et al., 1992; Kawada et al., 2001; Fiuza et al., 2004; Chia et al., 2010; Liu et al., 2013; Sun et al., 2014; Zhang et al., 2019). In addition to its high antioxidant activity; catechin was reported to exhibit several activities such as anti-inflammatory, antipandemic, anti-metastatic, anti-ulcer, anti-angiogenic, anti-allergic, anti-mutagenic, anti-proliferative, antimicrobial effects (Nagabhushan and Bhide, 1988; Maeda-Yamamoto et al., 2007; Xu et al., 2008; Chunmei et al., 2010; Delgado et al., 2014; You et al., 2018; Nakano et al., 2019). It was reported that p-coumaric acid exhibited immunomodulatory activity, antioxidant, antiinflammatory and antiangiogenic properties (Etoh et al., 2004; Kong et al., 2013; Pragasam et al., 2013). In previous studies, syringic acid was reported to possess hepatoprotective, anti-steatotic and anti-inflammatory effects (Itoh et al., 2009; Ham et al., 2016). In addition, it was evidenced that protocatechuic acid exhibited antiinflammatory, analgesic, anti-aging, anti-glycative, antiapoptotic, anti-influenza virus activities and anti-diabetic property (Lu et al., 2002; Min et al. , 2010; Lende et al., 2011; Tsai and Yin, 2012; Deng et al., 2014; Erukainure et al., 2017). Phenolic contents of $M$. melaleuca have not been previously determined. In a study on different species, it has been reported that Benzoic acid, pCoumaric acid, p-Hydroxybenzoic acid, Protocatechuic acid, Syringic acid and trans-Cinnamic acid are within Melanoleuca cognata (Fr.) Konrad and Maubl. and $M$. stridula (Fr.) Singer at different levels (Bahadori et al., 2019). In our study, p-coumaric acid, syringic acid and protocatechuic acid were determined in the body of $M$. melaleuca similar to these species. Unlike these species, it has been determined in Gallic acid and catechin in $M$. melaleuca. The present study findings suggested that $M$. melaleuca mushroom could be a natural source for these compounds.

Antioxidant and Oxidant Potential: Mushrooms synthesize various reduced molecules with antioxidant effects. In addition, they have the potential to contain antioxidant enzymes and coenzymes in their body. Furthermore, they are rich in vitamins A, C and E, which have antioxidant properties (Tan et al., 2018; Sevindik, 2018). TAS values are indicative of the system that mushrooms potentially produce in their structure and reflect the whole enzymatic and nonenzymatic molecules they possess (Selamoglu et al., 2020). Thus, determination of TAS is very important for the determination of new antioxidant natural resources. There are only a few studies in the literature that determined TAS, TOS and OSI of wild mushrooms. Furthermore, there are no studies that aimed to determine the TAS, TOS and OSI of $M$. melaleuca mushroom in the literature. In studies on edible mushrooms, TAS values for Cyclocybe cylindracea (DC.) Vizzini and Angelini,
Laetiporus sulphureus (Bull.) Murrill, Lentinus tigrinus (Bull.) Fr., Gyrodon lividus (Bull.) Sacc., Cerioporus varius (Pers.) Zmitr. and Kovalenko and Infundibulicybe geotropa (Bull.) Harmaja were reported as 4.325, 2.195, $1.748,2.077,2.312$ and $1.854 \mathrm{mmol} / \mathrm{L}$, respectively. TOS values for the same mushrooms were reported as 21.109 , $1,303,19.294,13,465,14.358$ and $30.385 \mu \mathrm{mol} / \mathrm{L}$, respectively. OSI values for the same mushrooms were reported as $0.488,0.059,1.106,0.651,0.627$ and 1.639 (Bal, 2018; Sevindik et al., 2018a; Sevindik, 2018; Sevindik et al., 2018b; Sevindik, 2019; Sevindik et al., 2020). In the present study, it was determined that the TAS of the $M$. melaleuca mushroom EtOH extract was $3.393 \pm 0.098 \mathrm{mmol} / \mathrm{L}$, TOS was $6.460 \pm 0.121 \mu \mathrm{mol} / \mathrm{L}$ and OSI was $0.190 \pm 0.002$. Thus, it was determined that the TAS value of $M$. melaleuca mushroom was lower when compared to that of $C$. cylindracea mushroom and higher when compared to that of L. sulphureus, L. tigrinus, $G$. lividus, C. varius and I. geotropa. It is suggested that these differences in TAS between the mushrooms were due to the differences in their capacity to produce antioxidant compounds. Furthermore, differences in the number and variety of secondary metabolites produced by mushroom defense system reactions to structural and environmental factors could have led to these findings. Thus, it was determined that M. melaleuca mushroom is suggested to be an important supplementary antioxidant nutrient.

In this study, it was determined that $M$. melaleuca mushroom TOS was lower than $C$. cylindracea, L. tigrinus, G. lividus, $C$. varius and $I$. geotropa mushrooms and higher than L. sulphureus mushroom. It is suggested that these differences were due to the differences between the regions where the mushrooms were collected, between the mushroom species, and between the substrates utilized by the mushrooms. Mushroom defense system mechanism stimulates the production of certain free radicals such as reactive oxygen species to protect itself against harmful endogenous factors in metabolite processes, particularly due to the environmental factors; thus, leading to changes in total oxidant levels in the mushroom (Kozarski et al., 2015; Sevindik, 2018). Consumption of these mushrooms may lead to various physiological disorders in humans, who are among the consumers in the environmental ecosystem (Frey-Klett et al., 2011). Therefore, determination of the total oxidant values of the mushroom samples collected in the regions where the mushrooms are consumed is very important. In the present study, TOS of the $M$. melaleuca mushroom was determined. The present study findings demonstrated that the analyzed mushrooms exhibited normal oxidant compound levels.

The OSI reflects the inhibition rate of oxidant compounds by the endogenous antioxidant compounds in living organisms. An increase in OSI reflects a lower 
inhibition rate of the endogenous oxidant compounds by the endogenous antioxidant compounds in the mushroom (Selamoglu et al., 2020). In the present study, it was determined that M. melaleuca mushroom OSI was lower when compared to that of C. cylindracea, L. tigrinus, $G$. lividus, C. varius and I. geotropa mushrooms and higher when compared to that of $L$. sulphureus mushroom. Since the total antioxidant system of edible M. melaleuca mushroom was more potent and effective against oxidant compounds in the present study, OSI values were low. Thus, it was determined that there was no significant disadvantage based on the oxidant compounds in consumption of the mushroom.

Antimicrobial activity: Today, due to the overconsumption of antibiotics, the resistance levels of microorganisms to antibiotics have increased (Stewart et al., 2018). Therefore, identification of new antimicrobial agents became mandatory. Mushrooms are exposed to several microorganisms in their natural ecosystem (FreyKlett et al., 2011). This has led to the development of defense systems for survival. Several studies demonstrated that mushrooms have significant effects on microorganisms. In studies previous studies, researchers from different parts of the world such as Chile, Brazil, Japan, Macedonia, Uruguay, Thailand, Korea, Turkey and Spain reported that mushrooms have antimicrobial effects on different types of microorganisms (Venturini et al., 2008; Akyuz and Kirbag 2009; Doğan et al., 2013; Kim et al., 2018; Rosenberger et al., 2018; Srichanun et al., 2018; Angelini et al., 2019; Yamada et al., 2019). In the present study, the effects of M. melaleuca mushroom EtOH extracts on the tested microorganisms were investigated. The findings are presented in Table 2.

Table 2. Antimicrobial Activities of M. melaleuca.

\begin{tabular}{lccccccccc}
\hline & $\boldsymbol{A}$ & $\boldsymbol{B}$ & $\boldsymbol{C}$ & $\boldsymbol{D}$ & $\boldsymbol{E}$ & $\boldsymbol{F}$ & $\boldsymbol{G}$ & $\boldsymbol{H}$ & $\boldsymbol{I}$ \\
\hline EtOH & 400 & 400 & 200 & 25 & 25 & 25 & 50 & 50 & 50 \\
Ampicillin & 1.56 & 3.12 & 1.56 & 3.12 & 3.12 & - & - & - & - \\
Amikacin & - & - & - & 1.56 & 3.12 & 3.12 & - & - & - \\
Ciprofloksasin & 1.56 & 3.12 & 1.56 & 1.56 & 3.12 & 3.12 & - & - & - \\
Flukanazol & - & - & - & - & - & - & 3.12 & 3.12 & - \\
Amfoterisin B & - & - & - & - & - & - & 3.12 & 3.12 & 3.12 \\
\hline
\end{tabular}

$25,50,200,400 \mu \mathrm{g} / \mathrm{mL}$ extract concentration

A: S. aureus, B: S. aureus MRSA, C: E. faecalis, D: E. coli, E: P. aeruginosa, F: A. baumannii, G: C. albicans, H: C. glabrata, I: C. krusei

Literature review revealed no study on the antimicrobial potential of $M$. melaleuca mushroom. It was determined that the mushroom extracts used in the present study were effective on test microorganisms at $25-400 \mu \mathrm{g} / \mathrm{mL}$ concentrations. It was found that mushroom extracts were particularly effective at 25 $\mu \mathrm{g} / \mathrm{mL}$ concentration on gram negative E. coli, $P$. aeruginosa and $A$. baumannii bacteria. In recent years, especially gram-negative bacteria have become a health problem due to their resistance to antibiotics. Thus, mortality rates have increased in infections caused by resistant bacteria (Frey-Klett et al., 2011). Researchers and the pharmaceutical industry have focused on various natural products effective on resistant bacteria (Selamoglu et al., 2020). In this study, it was determined that $M$. melaleuca extracts displayed the highest impact on gram negative bacteria. Furthermore, mushroom extracts were effective on C. albicans, C. glabrata and $C$. krusei fungal strains at $50 \mu \mathrm{g} / \mathrm{mL}$ concentration. In addition, mushroom extracts were effective on $S$. aureus, $S$. aureus MRSA and E. faecalis bacteria at high concentrations $(200-400 \mu \mathrm{g} / \mathrm{mL})$. As a result, it was determined that M. melaleuca mushroom analyzed in the present study was highly effective on tested gram negative bacterial and fungal strains. Furthermore, the antimicrobial potential of M. melaleuca was determined for the first time in the present study.

Conclusion: In this study, antioxidant potential, phenolic contents and antimicrobial potential of M. melaleuca edible mushroom, were determined. It was found that the mushroom had antioxidant properties. Furthermore, 5 compounds including gallic acid, catechin, p-coumaric acid, syringic acid and protocatechuic acid were identified in the mushroom. Antimicrobial activity of the mushroom was higher against the tested gram-negative bacteria, and fungal strains. Thus, it was concluded that edible M. melaleuca mushroom is a natural functional nutrient with biological potential.

Acknowledgments: I would like to express thanks to Dr. Nady BRAIDY, Dr. Hasan AKGÜL and Dr. Ilgaz AKATA for their contribution to article preparation.

\section{REFERENCES}

Akyuz, M. and S. Kirbag (2009). Antimicrobial activity of Pleurotus eryngii var. ferulae grown on 
various agro-wastes. Eurasia. J. Biosci. 3(8): 5863.

Angelini, P., C. Girometta, B. Tirillini, S. Moretti, S. Covino, M. Cipriani, E. D’Ellena, G. Angeles, E. Federici, E. Savino, G. Cruciani and R. Venanzoni (2019). A comparative study of the antimicrobial and antioxidant activities of Inonotus hispidus fruit and their mycelia extracts. Int. J. Food Prop. 22(1): 768-783.

Arora, S., S. Goyal, J. Balani and S. Tandon (2013). Enhanced antiproliferative effects of aqueous extracts of some medicinal mushrooms on colon cancer cells. Int. J. Med. Mushrooms 15(3): 301314

Bal, C. (2018). A Study on antioxidant properties of Gyrodon lividus. Eurasia. J. Forest Sci. 6(2): 4043.

Bal, C., M. Sevindik, H. Akgul, and Z. Selamoglu (2019). Oxidative Stress index and Antioxidant Capacity of Lepista nuda Collected From Gaziantep/Turkey. Sigma 37(1): 1-5.

Bahadori, M.B., C. Sarikurkcu, O.U. Yalcin, M. Cengiz and H. Gungor (2019). Metal concentration, phenolics profiling, and antioxidant activity of two wild edible Melanoleuca mushrooms ( $M$. cognata and M. stridula). Microchem. J. 150: 104172.

Bauer, A.W., W.M. Kirby, J.C. Sherris and M. Turck (1966). Antibiotic susceptibility testing by a standardized single disk method. Am. J. Clin. Pathol. 45: 493-96.

Caponio, F., V. Alloggio and T. Gomes (1999). Phenolic Compounds of Virgin Olive Oil: Influence of Paste Preparation Techniques. Food Chem. 64: 203-209

Chen, H.P. and J.K. Liu (2017). Secondary Metabolites from Higher Fungi. Progress in the Chemistry of Organic Natural Products 106: 1-201.

Chia, Y.C., R. Rajbanshi, C. Calhoun and R.H. Chiu (2010). Anti-neoplastic effects of gallic acid, a major component of Toona sinensis leaf extract, on oral squamous carcinoma cells. Molecules 15(11): 8377-8389.

Chunmei, D., W. Jiabo, K. Weijun, P. Cheng and X. Xiaohe (2010). Investigation of anti-microbial activity of catechin on Escherichia coli growth by microcalorimetry. Environ. Toxicol. Phar. 30(3): 284-288.

CLSI (The Clinical and Laboratory Standards Institute). (2012) Antimicrobial Susceptibility Testing of Anaerobic Bacteria; Approved StandardEighth Edition (M11-A8).

Delgado, L., I. Fernandes, S. González-Manzano, V. de Freitas, N. Mateus and C. Santos-Buelga (2014). Anti-proliferative effects of quercetin and catechin metabolites. Food and Function 5(4): 797-803.

Deng, J.S., S.D. Lee, W.W. Kuo, M.J. Fan, Y.M. Lin, W.S. Hu, Y.C. Huang, B.K. Velmurugan, F.J. Tsai, C.H. Tsai and C.Y. Huang (2014). Antiapoptotic and pro-survival effect of protocatechuic acid on hypertensive hearts. Chem-Biol. Interact. 209: 77-84.

Doğan, H.H., R. Duman, B. Özkalp and S. Aydin (2013). Antimicrobial activities of some mushrooms in Turkey. Pharma. Biol. 51(6): 707-711.

Erel, O. (2004). A novel automated direct measurement method for total antioxidant capacity using a new generation, more stable ABTS radical cation. Clin. biochem. 37(4): 277- 285.

Erel, O. (2005). A new automated colorimetric method for measuring total oxidant status. Clin. biochem. 38(12): 1103-1111.

Erukainure, O.L., R.M. Hafizur, M.I. Choudhary, A. Adhikari, A.M. Mesaik, O. Atolani, P. Banerjee, R. Preissner, A. Muhammad and M.S. Islam (2017). Anti-diabetic effect of the ethyl acetate fraction of Clerodendrum volubile: protocatechuic acid suppresses phagocytic oxidative burst and modulates inflammatory cytokines. Biomed. Pharmacother. 86: 307-315.

Etoh, H., K. Murakami, T. Yogoh, H. Ishikawa, Y. Fukuyama and H. Tanaka (2004). Anti-oxidative compounds in barley tea. Biosci. Biotech. Biochem. 68(12): 2616-2618.

EUCAST (European Committee on Antimicrobial Susceptibility Testing). (2014). Breakpoint tables Fungal isolate for interpretation of MICs. 2014; Version 7.0

EUCAST (European Committee on Antimicrobial Susceptibility Testing). (2015). Breakpoint tables for Bacteria interpretation of MICs and zone diameters 2015; Version 5.0

Fiuza, S.M., C. Gomes, L.J. Teixeira, M.G. Da Cruz, M.N.D.S. Cordeiro, N. Milhazes, F. Borges and M.P.M. Marques (2004). Phenolic acid derivatives with potential anticancer properties-a structure-activity relationship study. Part 1: Methyl, propyl and octyl esters of caffeic and gallic acids. Bioorg. Med. Chem. 12(13): 35813589.

Frey-Klett, P., P. Burlinson, A. Deveau, M. Barret, M. Tarkka and A. Sarniguet (2011). Bacterialfungal interactions: hyphens between agricultural, clinical, environmental, and food microbiologists. Microbiol. Mol. Biol. Rev. 75(4): 583-609.

Ham, J.R., H.I. Lee, R.Y. Choi, M.O. Sim, K.I. Seo and M.K. Lee (2016). Anti-steatotic and antiinflammatory roles of syringic acid in high-fat 
diet-induced obese mice. Food and Function 7(2): 689-697.

Hindler, J., L. Hochstein and A. Howell (1992). Preparation of routine media and reagents used in antimicrobial susceptibility testing. Part 1. McFarland standards, p. 5.19.1-5.19.6. In H. D. Isenberg (ed) Clinical microbiology procedures handbook, 1. American Society for Microbiology, Washington, D.C.

Itoh, A., K. Isoda, M. Kondoh, M. Kawase, M. Kobayashi, M. Tamesada and K. Yagi (2009). Hepatoprotective effect of syringic acid and vanillic acid on concanavalin a-induced liver injury. Biol. Pharm. Bull. 32(7): 1215-1219.

Kawada, M., Y. Ohno, Y. Ri, T. Ikoma, H. Yuugetu, T. Asai, M. Watanabe, N. Yasuda, S. Akao, G. Takemura, S. Minatoguchi, K. Gotoh, H. Fujiwara and K. Fukuda (2001). Anti-tumor effect of gallic acid on LL-2 lung cancer cells transplanted in mice. Anti-cancer drugs 12(10): 847-852.

Kim, S.P., S.J. Lee, S.H. Nam and M. Friedman (2018). The composition of a bioprocessed shiitake (Lentinus edodes) mushroom mycelia and rice bran formulation and its antimicrobial effects against Salmonella enterica subsp. enterica serovar Typhimurium strain SL1344 in macrophage cells and in mice. BMC Complement. Altern. Med. 18(1): 322.

Kong, C.S., C.H. Jeong, J.S. Choi, K.J. Kim and J.W. Jeong (2013). Antiangiogenic effects of pcoumaric acid in human endothelial cells. Phytother. Res. 27(3): 317-323.

Kozarski, M., A. Klaus, D. Jakovljevic, N. Todorovic, J. Vunduk, P. Petrović, M. Niksic, M.M. Vrvic and L. Van Griensven (2015). Antioxidants of edible mushrooms. Molecules 20(10): 19489-19525.

Kroes, B.V., A.J.J. Van den Berg, H.Q. Van Ufford, H. Van Dijk and R.P. Labadie (1992). Antiinflammatory activity of gallic acid. Planta medica 58(06): 499-504.

Lende, A.B., A.D. Kshirsagar, A.D. Deshpande, M.M. Muley, R.R. Patil, P.A. Bafna and S.R. Naik (2011). Anti-inflammatory and analgesic activity of protocatechuic acid in rats and mice. Inflammopharmacology 19(5): 255.

Liu, J., J.F. Lu, J. Kan and C.H. Jin (2013). Synthesis of chitosan-gallic acid conjugate: Structure characterization and in vitro anti-diabetic potential. Int. J. Biol. Macromol. 62: 321-329.

Lu, F.J., S.N. Tseng, M.L. Li and S.R. Shih (2002). In vitro anti-influenza virus activity of synthetic humate analogues derived from protocatechuic acid. Arch. Virol. 147(2): 273-284.

Maeda-Yamamoto, M., K. Ema and I. Shibuichi (2007). In vitro and in vivo anti-allergic effects of 'benifuuki'green tea containing O-methylated catechin and ginger extract enhancement. Cytotechnology 55(2-3): 135.

Matuschek, E., D.F. Brown and G. Kahlmeter (2014). Development of the EUCAST disk diffusion antimicrobial susceptibility testing method and its implementation in routine microbiology laboratories. Clin Microbiol Infect. 20: 255-266.

Meng, X., H. Liang and L. Luo (2016). Antitumor polysaccharides from mushrooms: a review on the structural characteristics, antitumor mechanisms and immunomodulating activities. Carbohydr. Res. 424: 30-41.

Min, S.W., S.N. Ryu and D.H. Kim (2010). Antiinflammatory effects of black rice, cyanidin-3$O-\beta-D-g l y c o s i d e$, and its metabolites, cyanidin and protocatechuic acid. Int. Immunopharmacol. 10(8): 959-966.

Muszyńska, B., A. Grzywacz-Kisielewska, K. Kała and J. Gdula-Argasińska (2018). Anti-inflammatory properties of edible mushrooms: A review. Food Chem. 243: 373-381.

Nagabhushan, M. and S.V. Bhide (1988). Antimutagenicity of catechin against environmental mutagens. Mutagenesis 3(4): 293-296.

Nakano, E., D. Kamei, R. Murase, I. Taki, K. Karasawa, K. Fukuhara and S. Iwai (2019). Antiinflammatory effects of new catechin derivatives in a hapten-induced mouse contact dermatitis model. Eur. J. Pharmacol. 845: 40-47.

Nimse, S. B. and D. Pal (2015). Free radicals, natural antioxidants, and their reaction mechanisms. Rsc Advances 5(35): 27986-28006.

Nowacka, N., R. Nowak, M. Drozd, M. Olech, R. Los and A. Malm (2015). Antibacterial, antiradical potential and phenolic compounds of thirty-one polish mushrooms. PLoS One 10(10): e0140355.

Pham-Huy, L. A., H. He and C. Pham-Huy (2008). Free radicals, antioxidants in disease and health. Int. J. Biomed. Res. 4(2): 89.

Pragasam, S.J., V. Venkatesan and M. Rasool (2013). Immunomodulatory and anti-inflammatory effect of p-coumaric acid, a common dietary polyphenol on experimental inflammation in rats. Inflammation 36(1): 169-176.

Rahman, K. (2007). Studies on free radicals, antioxidants, and co-factors. Clinical interventions in aging 2(2): 219.

Rosenberger, M.G., R. Paulert and V. Cortez (2018). Studies of the Antimicrobial Activity of Mushrooms (Agaricales) from South America. Int. J. Med. Mushrooms 20(11): 1065-1074.

Selamoglu, Z., M. Sevindik, C. Bal, B. Ozaltun, İ. Sen and A. Pasdaran (2020). Antioxidant, antimicrobial and DNA protection activities of phenolic content of Tricholoma virgatum (Fr.) 
P.Kumm. Biointerface Res. Appl. Chem. 10(3): 5500-5506

Sevindik, M. (2018). Investigation of antioxidant/oxidant status and antimicrobial activities of Lentinus tigrinus. Adv. Pharmacol. Sci. 2018. https://doi.org/10.1155/2018/1718025

Sevindik, M., H. Akgul, M. Dogan, I. Akata and Z. Selamoglu (2018a). Determination of antioxidant, antimicrobial, DNA protective activity and heavy metals content of Laetiporus sulphureus. Fresenius Environ. Bull. 27(3): 1946-1952.

Sevindik, M., H. Akgul, C. Bal and Z. Selamoglu (2018b). Phenolic contents, oxidant/antioxidant potential and heavy metal levels in Cyclocybe cylindracea. Indian J. Pharm. Educ. 52(3): 437441.

Sevindik, M. (2019). The novel biological tests on various extracts of Cerioporus varius. Fresenius Environ. Bull. 28(5): 3713-3717.

Sevindik, M., H. Akgul, Z. Selamoglu and N. Braidy (2020). Antioxidant and Antigenotoxic Potential of Infundibulicybe geotropa Mushroom Collected from Northwestern Turkey. Oxid. Med. Cell. Longev. 2020. https://doi.org/10.1155/2020/5620484

Srichanun, M., T. Lerssutthichawal, T. Nganwisuthiphan, and N. Chirapongsatonkul (2018). Possibility of split mushroom Schizophyllum commune byproduct extracts as antimicrobial and antioxidant agent for aquaculture. Int. J. Agri. Tech. 14(7 Special Issue): 1969-1976.

Stewart, C.A., Y. Finer and B.D. Hatton (2018). Drug self-assembly for synthesis of highly-loaded antimicrobial drug-silica particles. Scientific reports $8(1): 895$.

Suleman, M., A. Khan, A. Baqi, M.S. Kakar and M. Ayub (2019). 2. Antioxidants, its role in preventing free radicals and infectious diseases in human body. Pure Appl. Biol. 8(1): 380-388.

Sun, X., Z. Wang, H. Kadouh and K. Zhou (2014). The antimicrobial, mechanical, physical and structural properties of chitosan-gallic acid films. Lwt-Food Sci. Technol. 57(1): 83-89.

Tan, B. L., Norhaizan, M. E. and H. S. Winnie-Pui-Pui Liew (2018). Antioxidant and oxidative stress: A mutual interplay in age-related diseases. Front. Pharmacol. 16: 9 10.3389/fphar.2018.01162

Tsai, S. J. and M.C. Yin (2012). Anti-glycative and antiinflammatory effects of protocatechuic acid in brain of mice treated by D-galactose. Food Chem. Toxicol. 50(9): 3198-3205.

Venturini, M.E., C.S. Rivera, C. Gonzalez and D. Blanco (2008). Antimicrobial activity of extracts of edible wild and cultivated mushrooms against foodborne bacterial strains. J. Food Prot. 71(8): 1701-1706.

Xu, H., W.T. Lui, C.Y. Chu, P.S. Ng, C.C. Wang and M.S. Rogers (2008). Anti-angiogenic effects of green tea catechin on an experimental endometriosis mouse model. Human Reproduction 24(3): 608-618.

Yamada, S., M. Tanaka, R. Miura, C. Takeuchi, Z. Tu, D. $\mathrm{Hu}, \mathrm{K}$. Matsuoka, R. Sugawara, T. Hoshiba and A. Yamaguchi (2019). Anti-Inflammatory and Antimicrobial Activities of Aqueous Extracts of Wild Mushrooms from Japan. Int. J. Med. Mushrooms 21(5): 469-486

Yılmaz, A., S. Yıldız, S. Tabbouche, A.O. Kılıç and Z. Can (2016). Total phenolic content, antioxidant and antimicrobial properties of Pleurotus ostreatus grown on lime (Tilia Tomentosa) leaves. Hacettepe J. Biol. Chem. 44(2): 119-124.

You, H.L., C.C. Huang, C.J. Chen, C.C. Chang, P.L. Liao and S.T. Huang (2018). Anti-pandemic influenza A (H1N1) virus potential of catechin and gallic acid. Chin. Med. J. 81(5): 458-468.

Zanwar, A.A., S.L. Badole, P.S. Shende, M.V. Hegde and S.L. Bodhankar (2014). Role of gallic acid in cardiovascular disorders. In Polyphenols in human health and disease (pp. 1045-1047). Academic Press.

Zhang, X., J. Liu, C. Qian, J. Kan and C. Jin (2019). Effect of grafting method on the physical property and antioxidant potential of chitosan film functionalized with gallic acid. Food hydrocolloids 89: 1-10.

Zhong, J.J. and J.H. Xiao (2009). Secondary Metabolites from Higher Fungi: Discovery, Bioactivity, and Bioproduction. Biotechnology in China I: From Bioreaction to Bioseparation and Bioremediation 113: 79 . 